\title{
A Modified Color Averaging Technique for Content based Image Retrieval
}

\author{
Prabhakar Sharma \\ Department of Computer Science and Engineering \\ Chhatrapati Shivaji Institute of Technology, Durg, \\ C.G., India
}

\author{
Deepty Dubey \\ Department of Computer Science and Engineering \\ Chhatrapati Shivaji Institute of Technology, Durg, \\ C.G., India
}

\begin{abstract}
Content based image retrieval CBIR has been one of the most important research area in the field of computer science. There were many CBIR techniques have been proposed in the last decade. In this paper we propose a CBIR method which is modified form of color averaging technique. In previous color averaging techniques e.g. row mean, column mean ,forward diagonal mean, backward diagonal mean, sum of row ,columns and diagonal intensity values respectively were calculated for similarity measure. However it was possible that whether the sum could been same, but intensity values were not same for same corresponding positions in both query image and database image, as a result irrelevant images could be retrieved. In this method, we have tried to overcome this problem by considering the position of intensity values present in both query image and database images for similarity measure.
\end{abstract}

\section{Keywords - CBIR, Euclidean Distance}

\section{INTRODUCTION}

In literature the term content based image retrieval (CBIR) has been used for the first time by Kato et.al. [5], to describe his experiments into automatic retrieval of images from a database by color and shape feature[3].CBIR is an exciting and in-depth area of research, which has garnered much interest over the past few years [1]. Application of World Wide Web (www) and the internet is increasing exponentially, and with it the amount of digital image data accessible to the users. A huge amount of image databases are added every minute and so is the need for effective and efficient image retrieval systems [2]. The relevance of visual information retrieval in many areas such as fashion and design, crime prevention, medicine, law, and science makes this research field one of the important and fastest growing in information technology. Image retrieval has come a long way where it started off with text based retrieval. However, there are many problems associated with retrieving images based on text such as manual annotation of keywords, differences in perception and interpretations, and few others. Due to this researchers came up with CBIR where images are retrieved based on low-level features (human vision related), middle-level features (objects related), or low-level features (semantic related). Among these features low-level features are the most popular due to its simplicity compared to other level of features plus automatic object recognition and classification is still among most difficult problems in image understanding and computer vision [1]. The low-level features are color, texture, shape, and spatial properties. However spatial properties are implicitly taken into account so the main features to investigate are color, texture and shape. Color feature is one of the most widely used features in low level feature [6]. Compared with shape feature and texture feature, color feature shows better stability and is more insensitive to the rotation and zoom of image. Color not only adds beauty to objects but also more information [7]. Texture generally refers to the presence of a spatial pattern that has some properties of homogeneity. Directional features are extracted to capture image texture information. The four extraction methods available for texture feature retrieval are The Steerable Pyramid, The Contour let Transform, The Gabor wavelet Transform, and The Complex Directional Filter Bank[8].

\section{PREVIOUS WORK}

A method proposed by Dr. H.B.Kekre, Sudeep D. Thepade, Akshay Maloo all image pixels are considered as feature vector and Euclidean distance is used in RGB plane to find the best match, which is used to calculate precision and recall. Another method proposed by Dr. H.B.Kekre, Sudeep D. Thepade, and Akshay Maloo row mean of image is calculated to be feature vector and then Euclidean distance is used in RGB plane to find the best match, which is used to calculate precision and recall. In column method proposed by Dr. H.B.Kekre, Sudeep D. Thepade, and Akshay Maloo feature vector is composed of column mean of image is calculated and then Euclidean distance is used in RGB plane to find the best match, which is used to calculate precision and recall. In row and column mean of image method proposed by Dr. H.B.Kekre, Sudeep D. Thepade, Akshay Maloo row and column mean of image are considered together as feature vector and then Euclidean distance is used in RGB plane to find the best match, which is used to calculate precision and recall. In forward diagonal method, backward diagonal mean of image is considered as feature vector and then Euclidean distance is used in RGB plane to find the best match, which is used to calculate precision and recall. In forward diagonal method, forward diagonal mean of image is calculated and then Euclidean distance is used in RGB plane to find the best match, which is used to calculate precision and recall. Both methods were proposed by Dr. H.B.Kekre, Sudeep D. Thepade, Akshay Maloo. They also purposed another method in which both forward and backward diagonal mean both are considered together as feature vector of image and then Euclidean distance is used in RGB plane to find the best match, which is used to calculate precision and recall[3][4].

\section{PROPOSED METHOD}

The concept behind this proposed method is that if two images are very relevant then in both images at same positions there will be same or very nearest intensity values will present. 


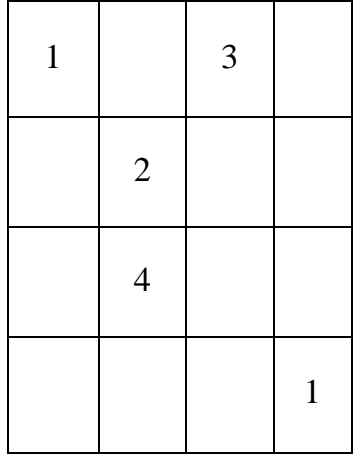

a) Query Image

\begin{tabular}{|l|l|l|l|}
\hline 1 & & 3 & \\
\hline & 2 & & \\
\hline & 3 & & \\
\hline & & & 1 \\
\hline
\end{tabular}

b) Database Image
Fig. 1 figure shows Query image and Database Image

Fig.1 (a) and fig.1 (b) shows query and database image respectively, where 1,2,3,4 shows intensity value of pixels present in both query image and database image with corresponding positions. In this method, calculations are based on position of specific intensity values present in both query image and database image. In this method we have to first find out the intensity values present in query image with their respective positions in query image. In the next Step for each intensity values present in query image we calculate average of intensity values present in corresponding positions in database image. To perform this calculation it is required to have both images should be of same size. The steps of algorithms are as follows .

Step1: Find out the position of intensity values present in query image. Because digital images are represented in the form of matrix, it is difficult to find the location of any intensity values present in image. To resolve this problem we need to represent the image into vector form. In this representation we can easily find the location of an intensity value present in query image.

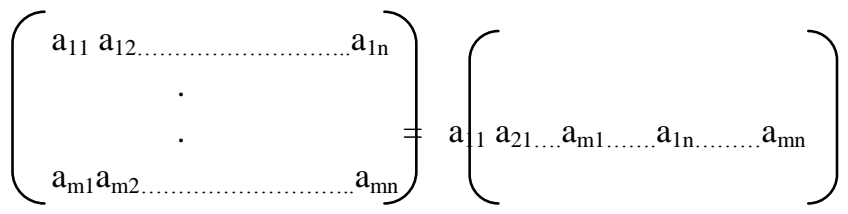
(a) Digital image in Matrix form
(b) Digital image in vector form

Fig. 2: Digital image in matrix form and Digital image in vector form

Fig.2 shows the matrix and vector representation of digital image, in which $\mathrm{a}_{11}, \mathrm{a}_{12} \ldots \mathrm{a}_{1 \mathrm{n}} \ldots \mathrm{a}_{\mathrm{m} 1}, \mathrm{a}_{\mathrm{m} 2} \ldots \mathrm{a}_{\mathrm{mn}}$ are intensity value of pixels present in digital image.
Step 2: Find the average of intensity values present in database image for corresponding positions of a intensity value in query image. To perform this operation, it is require representing the database image in vector form. In this way we can easily locate the positions of intensity vales found in query image into database image. But before performing this operation it is necessary to make the database image size same as query image size. For example

\begin{tabular}{|l|l|l|}
\hline 1 & 2 & 3 \\
\hline 1 & 2 & 1 \\
\hline 3 & 1 & 2 \\
\hline
\end{tabular}

a) Query image

\begin{tabular}{|l|l|l|}
\hline 1 & 3 & 2 \\
\hline 2 & 1 & 1 \\
\hline 3 & 1 & 2 \\
\hline
\end{tabular}

b) Database Image
Fig. 3: Query image and Database image

For intensity value ' 1 ' in query image positions are $1,2,6,8$.For this corresponding positions in database image, average of intensities will be

$$
\frac{1+2+1+1=5}{4}=\frac{}{4} 1.25
$$

Step 3: Perform the similarity measure between query image and database image by taking difference of a intensity value in query image and average intensity for that intensity in database image. This similarity measure is performed using Euclidean Distance formula

$$
D=\sqrt{\sum_{i=1}^{N}\left({ }^{N p i-V q i}\right)^{2}}
$$

Equation (1), calculates the Euclidian distance between two pixel values, where, Vpi and Vqi are the feature vectors of image $\mathrm{P}$ and Query image $\mathrm{Q}$ respectively with size ' $\mathrm{N}$ '.

Step 4: Retrieve those images for which Euclidean distance is minimum. 


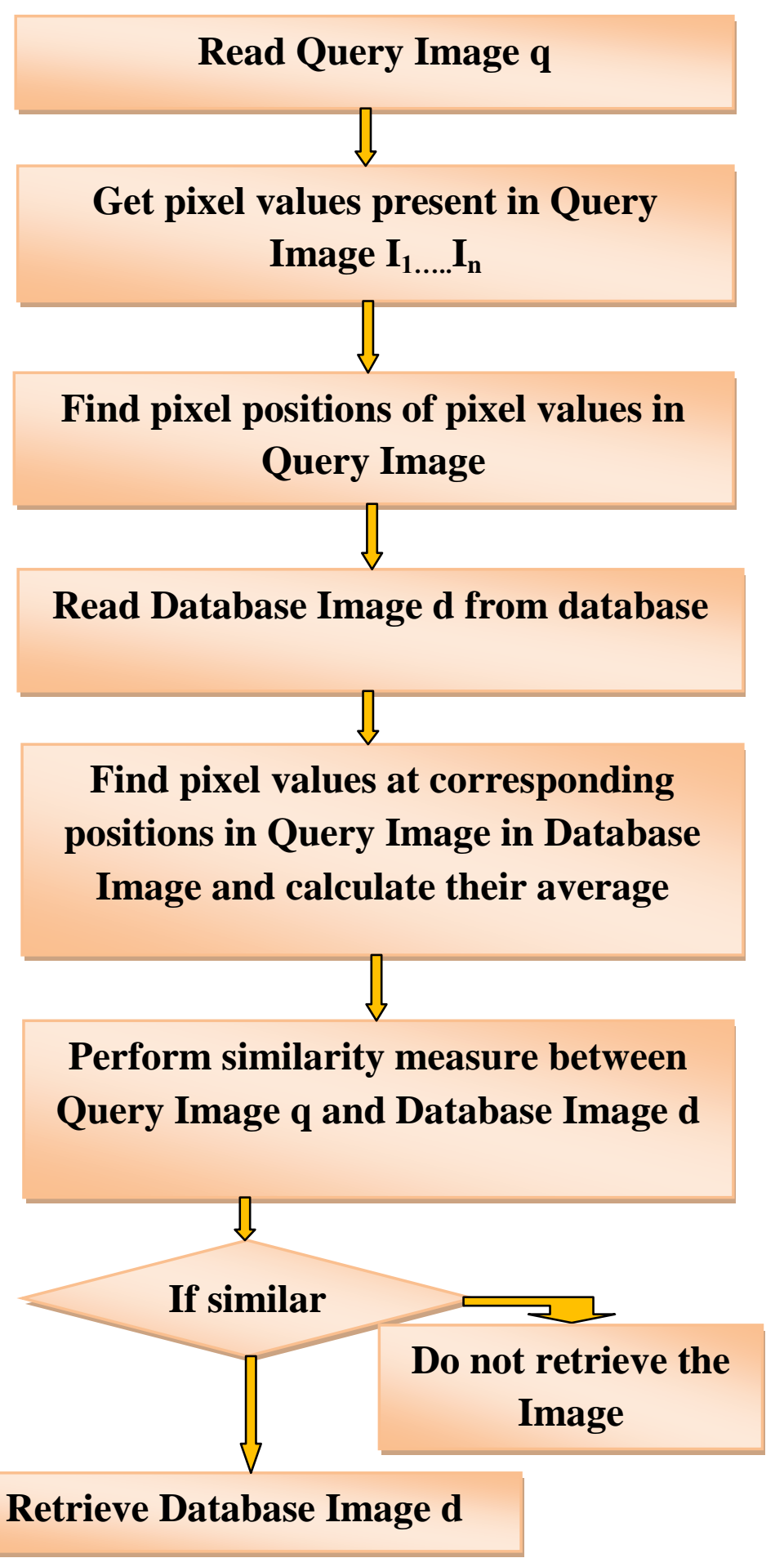

Fig. 4: Flow diagram

\section{RESULT ANALYSIS}

In database we have 1000 images which is categorized in 10 classes. Some of these images are shown in fig. 5. Fig. 6 shows the expected result after a query image is selected for finding its best match.

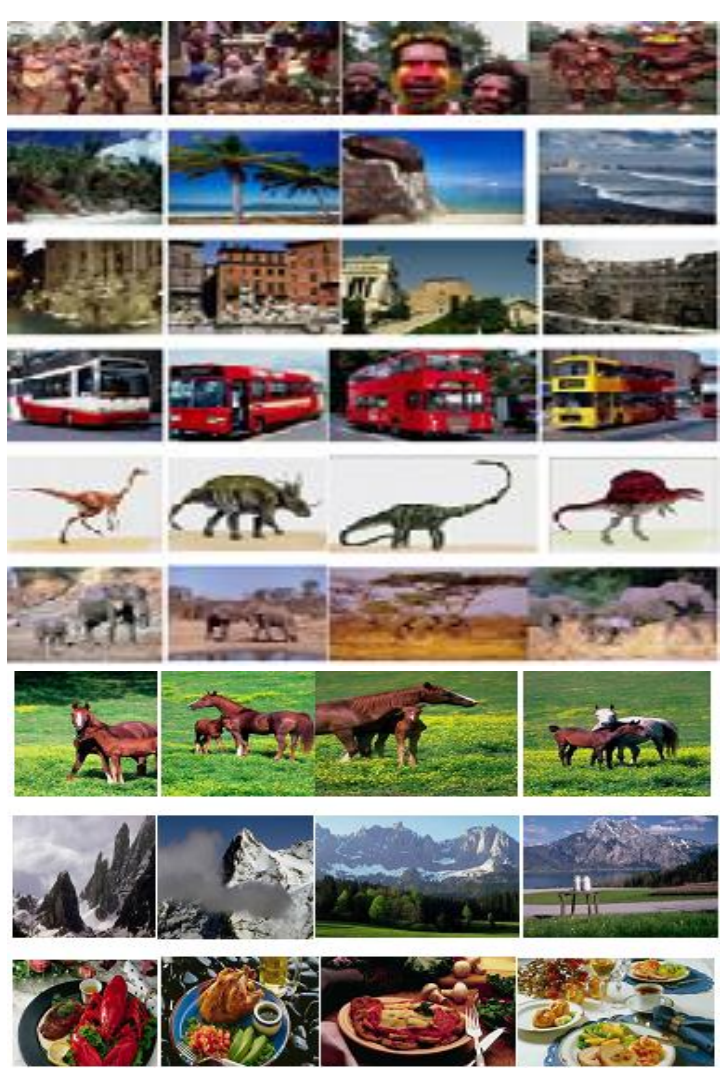

Fig. 5: Database images

From proposed method, we expect the following results.

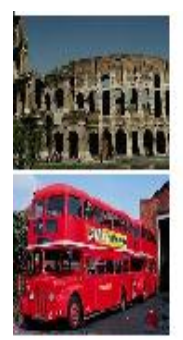

Query image

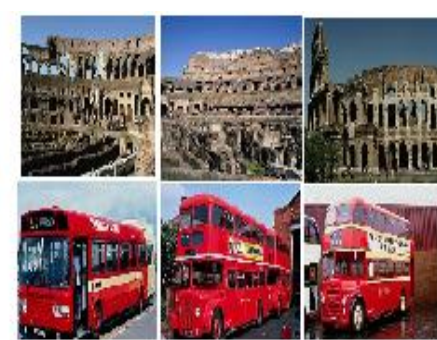

Retrieved Image
Fig. 6: Expected Result

\section{CONCLUSION}

In this paper we have proposed a modified retrieval technique using color averaging method based on pixel positions. In another color averaging technique it is possible that for same positions in query image and database image, there might have different pixel intensities. So it is possible that retrieved images will not be very similar to query image. But in our proposed method, we performed similarity measure between pixel intensities present in both query image and database image for same positions. Hence the possibility of retrieving images similar to query image may increase. The proposed method may be useful for colorization of grayscale image, where best match of source image pixels is must. 


\section{REFERENCES}

[1] Mas Rina Mustaffa, Fatimah Ahmad, Rahmita Wirza O.K. Rahmat, Ramlan Mahmod." Content-based image retrieval based on color-spatial features" Malaysian Journal of Computer Science, Vol. 21(1), 2008.

[2] P. V. N. Reddy, K. Satya Prasad, " Color and Texture Features for Content Based Image Retrieval”, P V N Reddy et al, Int. J. Comp. Tech. Appl., Vol 2 (4), 1016-1020.

[3] Dr. H.B.Kekre ${ }^{1}$, Sudeep D. Thepade ${ }^{2}$, Akshay Maloo,", Query by Image Content Using Color Averaging Techniques", Dr. H.B. Kekre et al. / International Journal of Engineering Science and Technology.Vol. 2(6), 2010, 1612-1622.

[4] Dr. H.B.Kekre, Sudeep D. Thepade, Akshay Maloo," Image Tiling to Improve Performance of Image Retrieval Using Color Averaging Techniques", IJCA Special Issue on "Computer Aided Soft Computing Techniques for Imaging and Biomedical Applications" CASCT, 2010
[5] Hirata K. and Kato T. "Query by visual example - contentbased image retrieval", In Proc. of Third International Conference on Extending Database Technology, EDBT'92, 1992, pp 56-71

[6] Th.Gevers (2001). "Color Based Image Retriev-al" Springer Verlag GmbH. pp.886-917

[7] Manesh Kokare, B.N. Chatterji and P.K. Biswas, "A survey on current content based image retrieval methods", IETE Journal of Research, Vol. 48, No.3 and 4, May-Aug 2002.

[8] H.B.Kekre, Sudeep D. Thepade, "Rendering Futuristic Image Retrieval System", National Conference on Enhancements in Computer, Communication and Information Technology, EC2IT-2009, 20-21 Mar 2009, K.J.Somaiya College of Engineering, Vidyavihar, Mumbai-77. 